\title{
Exploring Urban Interventions through Computational tools: genetic algorithm and urban connection patterns
}

\section{SIGRADI2018 TECHNOPOLITICAS \\ xxii congresso da sociedade iberoamericana de gráfica digital 22th conference of the iberoamerican society of digital graphics 07|08|09|novembro|2018 iau usp | são carlos | sp br}

\author{
Manoel Rodrigues Alves \\ Instituto de Arquitetura e Urbanismo: Universidade de São Paulo | Brasil | mra@sc.usp.br
}

\section{Alvaro Martins Abdalla}

Escola de Engenharia São Carlos: Universidade de São Paulo | Brasil | pfalvaro@sc.usp.br

Carlos Tapia

Escuela Técnica Superior de Arquitectura: Universidad de Sevilla | Espanha | tava@us.es

\begin{abstract}
This paper presents a particular approach to design processes in urban design, in a transdisciplinary environment. Exploring geotechnologies, information and communication technologies, artificial intelligence techniques and experimental softwares (fuzzy logic and generic algorithm), the workshop "Generation of Urban Connection Patterns", developed by IAU-USP (Brazil) and ETSA-US (Spain), aimed: to investigate urban space connection patterns in areas of environmental and social vulnerability; to explore formal arrangements in urban design; to foster academic exchange and possibilities of collaborative workshops. The article also discusses the role of computational tools and the implementation of in-person and non-presential methods in the teaching/learning process.
\end{abstract}

Keywords: Transdisciplinarity; Teaching and Learning; Genetic Algorithm; Urban Connection Patterns; Urban Design.

\section{INTRODUÇÃO}

Explorando técnicas de inteligência artificial, ferramenta de tecnologia da informação e comunicação e o emprego de geotecnologias e de softwares experimentais (lógica fuzzy e algoritmo genético), o workshop projetual "Geração de Padrões Urbanos de Conexão", desenvolvido em disciplina de graduação do Instituto de Arquitetura e Urbanismo da Universidade de São Paulo (IAU-USP, Brasil) e da Escuela Técnica Superior de Arquitectura da Universidad de Sevilla (ETSA-US, Espanha), investigou processos e procedimentos projetuais em arquitetura e urbanismo objetivando: a exploração projetual de propostas urbanas em ambiente transdisciplinar colaborativo; o desenvolvimento de padrões urbanos, morfológicos e formais, de conexão entre diferentes estruturas urbanas; a exploração de parâmetros urbanísticos na otimização de arranjos formais; potencializar o intercâmbio acadêmico, discente e docente.

Problematizando aspectos da prática pedagógica no campo da arquitetura e do urbanismo, em seu início, o artigo argumenta da importância de se explorar possibilidades alternativas para a formação de arquitetos, Após contextualizar e descrever a atividade didática e a metodologia adotada, o texto, em sua parte final, analisa limites e potencialidades das práticas e experimentações do workshop, em particular do ferramental computacional empregado e dos processos e procedimentos projetuais adotados na implementação de métodos de ensino / aprendizagem presenciais e não-presenciais.

\section{CONTEXTUALIZAÇÃO}

Em grande medida, boa parte das proposições relativas a práticas pedagógicas e metodologias didáticas que se encontram na literatura sobre o ensino de arquitetura e urbanismo não promovem uma reflexão integral sobre a formação do arquiteto. Revistas e congressos publicam, como alternativas, práticas docentes que, se limitando a exemplificar o exercício da arquitetura e do urbanismo, não parecem comprender, ou querer diagnosticar o que acontece em nosso tempo. Uma breve revisão, desde os finais do século XIX até nossos dias, pode constatar a permanente falta de correspondência entre o que a arquitetura faz e a sociedade que a alberga, fato esse que se desdobra em uma formação (cursos) que mais parece asumir o papel de centros de adestramento de arquitetos.

Assim o explicam, por exemplo, grandes figuras que contribuíram na construção do meio acadêmico em sua sociedade, como Jean Labatut e Robert Gutman em Princeton, Luis Moya em Madrid, Darcy Ribeiro em Brasilia, John Hedjuk na Cooper Union, ou ainda Alvin Boyarski na Architectural Association - cujo plano de estudos dos anos 50 do século passado se diferenciava do modelo de ensino da Bauhaus pela estrutura hierárquica de "cápsulas" ordenadas pela amplitude de unidades temáticas (casa, quadra, equipamento público, industrial, bairro, cidade).

Kenneth Frampton (Mitásová, 2014) qualifica essa aproximação de Boyarski como básica para um ensinar que se propunha a abrir caminhos e inaugurar novas práticas arquitetônicas, argumento esse que permanece nos projetos pedagógicos e planos de estudo de arquitetura da segunda década do século XXI. Zygmunt Bauman (Bauman, 2007) observa, em relação aos desafios que hoje se colocam, que o ensino, ao depararse com a "síndrome da impaciência" ( de uma aceleração de tudo a todo custo), meramente promove a 
aprendizagem do que deverá ser posto em prática na profissão - traduzindo, concretamente, objetivos docentes de semestres de 15/17 semanas.

Um modelo de ensino que, por não explorar possibilidades de métodos de ensino/aprendizagem, ratifica a opinião de Bauman quanto a obsolescência de práticas didáticas e pedagógicas empregadas para enfrentar os desafios da educação. Desafios esses que, observando educação enquanto forma de recuperar uma condição de conhecimento duradouro, caracterizam uma questão incerta: como preparar os estudantes de arquitetura a refletirem sobre esses processos (de aceleração) que condicionam, no mínimo impactam, transformações da sociedade e da noção de cidade, portanto, a prática profissional da arquitetura?

Observa-se, na formação do arquiteto e do urbanista, o marasmo de uma corrente imperante de propostas docentes de 'inovação' que se caracterizam pela 'profusão' mental de conhecimentos assimilados, antes mesmo de serem postos (e provados) em prática. De fato, via de regra, o ambiente de formação e os espaços de ensino/aprendizagem se constituem, como um todo, em um produto de consumo, ensimesmado e efêmero, em certos meios marcados pela imagem. Como então proceder o aprender a apreender, a compreender, a pensar, a assumir problemáticas e a propor a produção de arquitetura? Ou seja, refletir, dar a pensar antes que tecnificar em competências específicas.

Entendemos adequado e necessário explorar o desenvolvimento de ambiente colaborativo transdisciplinar de ensino / aprendizagem, enquanto processo e instrumento de produção do conhecimento. Nesse contexto, o workshop projetual "Geração de Padrões Urbanos de Conexão" buscou promover a instrumentalização dos estudantes no desenvolvimento de um olhar crítico na análise de problemáticas contemporâneas da cidade e do ambiente urbano - não só de sua leitura e apreensão, mas também de sua concepção e processos de desenho.

Essa abordagem decorre de entendimento de que a transdisciplinaridade, termo de Jean Piaget ao princípio dos anos 70, referente ao entendimento do mundo de hoje, pode possibilitar, em relação ao pensar e fazer arquitetônico, a construção de pontes, articulações entre ciências e humanidades, pensando dentro e entre a arte, a tecnologia, a ciência e a filosofia.

Para Nicolescu (Nicolescu, 2013), a transdisciplinaridade compreende três postulados: a existência de níveis de realidade, a lógica do tertium datur e a complexidade. A transdisciplinaridade aponta para a unidade do conhecimento, objetivando estimular uma compreensão da realidade que articule elementos que passam entre, ademais de uma busca de compreensão do mundo real. Portanto, não só significa que os conteúdos disciplinares (não necessariamente organizados em disciplinas) se articulam e colaboram entre si, mas que existe um pensamento organizador que sobrepassa as próprias matérias diferentemente: da multidisciplinaridade, conjunto de disciplinas trabalhadas simultaneamente que não promove as relações que possam existir entre elas, uma vez que condicionado por um sistema de um só nível e de objetivos únicos, sem cooperação; ou da interdisciplinaridade, que apesar de promover um certo nível de agrupamento e justaposição de matérias/disciplinas e, portanto, expressar um certo grau de relações entre elas, constitui-se enquanto sistema de disciplinas de um só nível, de uma mesma hierarquia. No contexto desse entendimento, e considerando que a transdisciplinaridade demanda um pensamento organizador, o workshop buscou aplicar reflexivamente a noção de transdisciplinaridade de modo a explorar práticas alternativas para o ensino projetual no campo da Arquitetura e do Urbanismo.

Tendo como referência a problematização de uma cidade que se caracteriza mais pela intensidade do que pela densidade, em que se detecta um interesse particular por sua delimitação final, sua 'urban fringe', o workshop se estruturou na pergunta: é possível definir o limite da cidade ou estabelecer padrões de articulação do espaço urbano? Pergunta essa que em sua problematização, em função da área de trabalho e do perfil dos alunos, considerou também que na cidade observam-se distinções e singularidades entre o caso europeu, em que as estruturas medievais se ressentem do encontro com as inserções fabris, e o latinoamericano, em que o espraiamento e a expansão urbana caracterizam um processo de periferialização. Em ambos os casos, a expansão das cidades e/ou a obsolescência de suas estruturas urbanas, geraram distintos tipos de vazios urbanos, físicos e sociais, que condicionam uma cidade de tecidos e estruturas urbanas e bolsões desarticulados; uma cidade em que efeitos da metropolização, de distintas escalas, impactam e demandam a revisão de desenvolvimento de propostas urbanas e de seus processos.

Trata-se não apenas de superar dogmas do ensino projetual, mas também de explorar os limites do projeto urbano na superação de um urbanismo fracassado de planos de longo prazo. De explorar um desenho urbano que (na acepção do termo urban design) dinâmico e aberto a redefinição de processos que, por meio de uma sucessão de ações projetuais imaginadas (e não definidas desde o princípio), potencializem interesses singulares. No workshop, a exploração paramétrica de propostas urbanas, que podem possibilitar a construção de um outro enfoque que considere o desenho urbano não como um produto derivado exclusivamente da forma, função ou do meio ambiente, mas sim como um produto das relações entre os diversos elementos de um sistema mais complexo que é a própria cidade.

Patrick Schumacher, obstinado em dar sentido ao que chama parametricismo, aporta questões que, por um lado, ilustram a complexidade dos procedimentos arquitetônicos, mas, por outro, não vão além, não superam o debate pelo estilo. Se o herdeiro do estudio Zaha Hadid Architects quer estabelecer uma suposta via imutável da arquitetura, que culmina com a evolução estilística dos patterns, assemelhando esta operação a uma Modernidade do século XXI, haverá de justificar esse argumento mais claramente apesar da imensa quantidade de artigos em que investe para isso. Os parâmetros da forma, da função e do meio ambiente devem estar interrelacionados aos parâmetros de configuração do espaço.

O Urbanismo Paramétrico pode também auxiliar na construção de outras lógicas de desenho urbano que explorem novas técnicas de variação e diferenciação formal - por exemplo, por meio de técnicas de deformação como geometrias curvas complexas de linhas splines e superficies NURBS. Lógicas exploratórias de tramas de articulação do tecido urbano de novos projetos aos tecidos 
preexistentes que investiguem padrões e possibilidades de conexão da trama urbana em seu conjunto.

Investigando a pertinência de definição dos limites, das bordas da cidade e do estabelecimento de padrões de articulação do espaço urbano, o workshop "Geração de Padrões Urbanos de Conexão", trabalhou conteúdos da arquitetura e do urbanismo, explorando noções da geomática e da aeronáutica na investigação projetual. Para tanto, explorou a transdisciplinaridade na experimentação de processos de ensino/aprendizagem e procedimentos projetuais parametricizados (em arquitetura e urbanismo) por meio do emprego de técnicas de inteligência artificial (IA) e tecnologia da informação e comunicação (TIC) na aplicação conjunta de: Entropia Estatística (EE); Desdobramento da Função de Qualidade (QFD); Lógica Fuzzy (LF); e Algoritmo Genético (AG), associado ao uso de geotecnologias e a utilização de modelador de sólidos.

De fato, problematizando a geração de padrões de conexão entre tecidos urbanos distintos nas 'bordas' da cidade, promovendo a interpretação de dados de Big Data mediante o uso de App para Android MAPinr, aplicando algoritmos genéticos e refletindo sobre chaves da contemporaneidade por meio do emprego de metodologia de projetação que permite o controle do processo e a modelação de resultados - mas não, em absoluto, a "automatização" ou o "engessamento" das soluções de projeto -, se aportou aos estudantes uma visão estimulante, do que possa ser hoje entendido e praticado na investigação de soluções para um problema urbano.

No caso, o desenvolvimento de propostas para a rearticulação de tecidos urbanos distintos, situados em área de vulnerabilidade social e ambiental da cidade de Sevilha, na borda oeste da Ilha da Cartuja. Os processos de experimentação projetual compreenderam três fases: uma, de formulação de requisitos e especificações de projeto referentes a critérios, parâmetros e variáveis arquitetônicas (condições de contorno por meio de lógica Fuzzy e entropia estatística); outra conceitual, de avaliação do desenvolvimento do projeto e dos parâmetros empregados no algoritmo genético; e uma terceira de análise das soluções e definição de diretrizes de desenvolvimento com a eventual redefinição dos parâmetros urbanísticos.

Estudos recentes identificam subcategorias arquitetônicas decorrentes da investigação com diferentes técnicas de geração digital. São arquiteturas digitais estruturadas com base nos conceitos de: espaço topológico (arquitetura topológica); superficies isomorfas (arquitetura isomorfa); cinemática e dinâmica de movimento (arquitetura animada); animação (arquitetura metamórfica); desenho paramétrico (arquitetura paramétrica); e algoritmos genéticos (arquitetura evolutiva, capacidade de transformação). O workshop explorou, principalmente, o último dos conceitos, associado a procedimentos de parametrização. Um algoritmo genético é a aplicação dos conceitos da teoría de evolução darwiniana em um meio informacional. Do mesmo modo que a natureza gera, seleciona e combina os indivíduos com o fim de gerar mais probabilidades de sobreviver, o emprego de um algoritmo genético comprende a seleção e combinação de alternativas para a otimização da solução de um problema. Essa possibilidade foi explorada por uma série de passos, guiados por parámetros pré-determinados, por meio do emprego de software de otimização para soluções formais parametricizadas: o Kaipira. Associado ao emprego de software de modelação de sólidos (SolidWorks), o Kaipira possibilitou o refinamento contínuo das soluções projetuais a partir da eventual revisão, a criterio de cada grupo de trabalho, dos parâmetros de iteração e urbanísticos empregados.

\section{METODOLOGIA}

O workshop possibilitou a experimentação de métodos inovadores no desenvolvimento projetos urbanos, considerando que a aplicação do ferramental computacional deve ser entendido como meio, e não como um fim em si mesmo (reflexividade transdisciplinar). Introduzindo os alunos a processos projetuais, até certo ponto experimentais, o workshop investigou procedimentos de ensino / aprendizagem que possibilitassem a exploração de práticas não usuais no desenvolvimento de soluções de projeto.

Com o objetivo de potencializar a aquisição e produção de conhecimento, os estudantes foram convidados, em um ambiente de experimentação de abordagens teóricas e ferramentas multimeios, a empregarem formas nãolineares de construção e apresentação de suas ideias, bem como a avaliar não apenas processos e procedimentos projetuais mas também, por meio de explorações formais topológicas, possíveis experimentações narrativas de linguagens arquitetônicas (ver Fig. 1).

O workshop contou com a participação de docentes das áreas de aeronáutica, arquitetura, geomática e urbanismo, os professores Alvaro Abdalla (EESC-USP), Manoel Rodrigues Alves e Marcel Fantin (IAU-USP) e Carlos Tapia (ETSA-US). As atividades didáticas, diversas vezes por videoconferência, compreenderam aulas expositivas, laboratoriais (capacitação dos alunos em ferramentas computacionais) e atividades de atelier (desenvolvimento de propostas, discussão e apresentação dos trabalhos),. Os trabalhos foram desenvolvidos por grupos de 6 alunos de ambos os cursos de arquitetura e urbanismo (3 de Sevilha e 3 de São Carlos), implicando tanto no desenvolvimento de atividades específicas em cada sede quanto em trabalho colaborativo em horários extraclasse, a critério de cada grupo.

O programa da disciplina compreendeu: 1. introdução e problematização do emprego de geotecnologias, explorações formais e variações paramétricas, lógica fuzzy, (LF) e Entropia Estatística (EE, associada a parametrização do projeto), Desdobramento da Função de Qualidade (QFD, na tradução de informação subjetiva e de requisitos para números reais) e Algoritmo Genético; 2. introdução ao Kaipira e exploração de possibilidades formais por meio de modeladores de sólidos; 3. processos projetuais, estudo de casos e exploração projetual em atelier; 4. em relação aos conteúdos teóricos, imaginário e paisagem da cidade contemporânea e aspectos do processo de urbanização de Sevilha e São Paulo, evolução morfológica e social. 


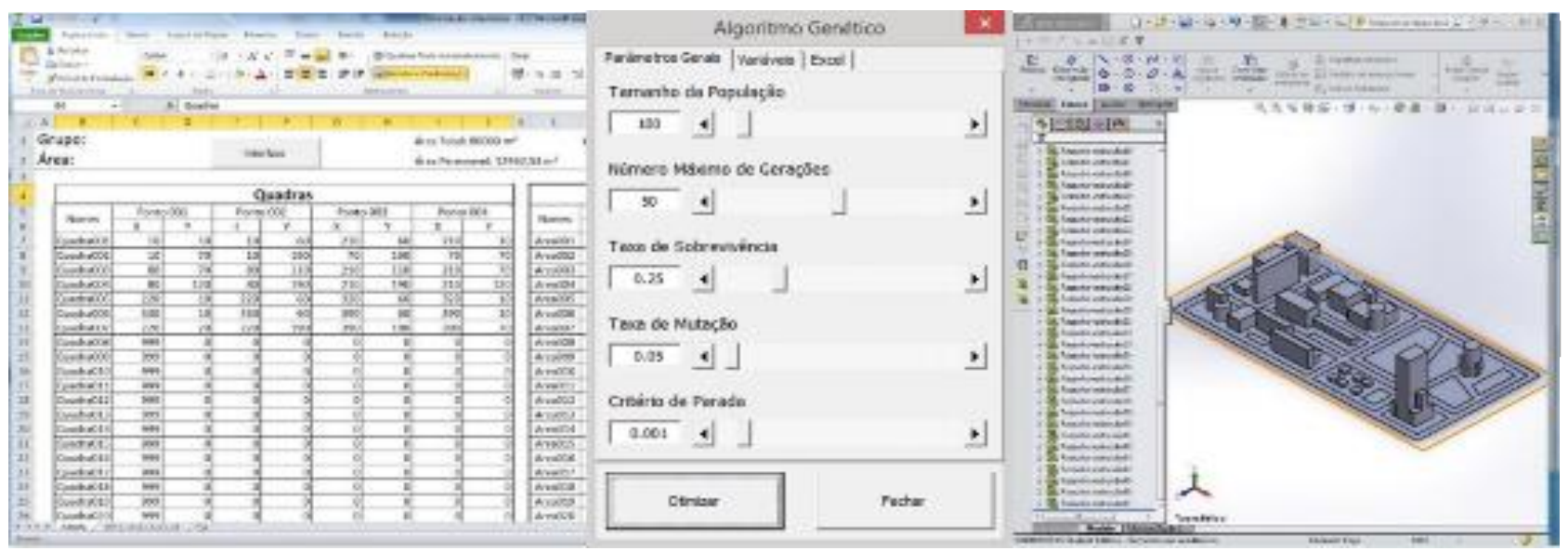

Figura 1: Tela de Cálculo do Algoritmo Genético do Caipira. Modelação tridimensional de proposta em desenvolvimento (SolidWorks).

$\mathrm{Na}$ construção da base de dados e na manipulação da informação espacial se adotor, em virtude de sua facilidade de manejo, requerer um menor tempo de capacitação e demandar apenas conhecimentos básicos dos estudantes na interpretação de imagens pancromáticas com 1.65 metros de resolução espacial do satélite Geo Eye, o software de Google Earth. O procedimento que se seguiu foi a conversão de arquivo georreferenciado de esri shapefile com formato $\mathrm{km} /$ Google e a incorporação desses arquivos na base de dados de modo a permitir, por meio da incorporação de dados georreferenciados (equipamentos e serviços públicos urbanos, hidrografía, áreas verdes, dentre outros), a superposição e análise de características da área de estudo em Sevilha.

Essa base de dados foi armazenada em formato $\mathrm{kml}$ e disponibilizada aos estudantes objetivando contribuir com a análise das diretrizes de projeto e o emprego de MAPinr (aplicativo de Android para atividades de campo), incluindo suporte GPS para a coleta, gravação e classificação de informações. Por meio de sua valoração, essas informações possibilitaram a produção de cartografia referente a segurança e conflitos potenciais, inserção e produção habitacional, problemas ambientais e padrão morfológico e a composição socioespacial e funcional do tecido urbano (ver Fig. 2).
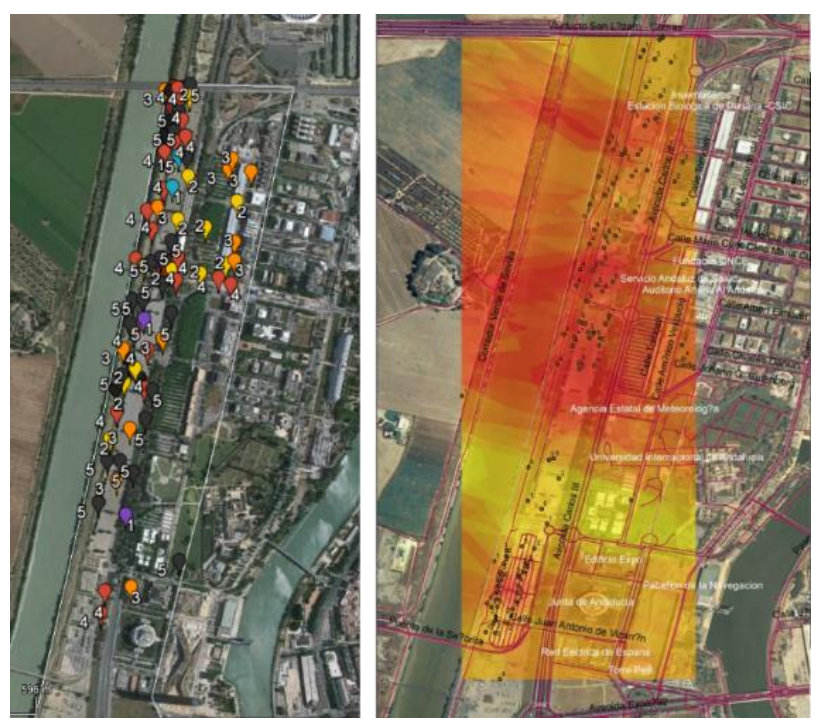

Figura 2: Dados georreferenciados, aplicação do MAPinr sobre Google Earth. Mapa de Preliminar de Potencialidades.
O Kaipira, ferramenta de simulação computacional desenvolvida em ambiente Microsoft Virtual Basic empregado na disciplina entende, na utilização dos dados para o desenvolvimento de possibilidades: heurística, como conjunto de regras e procedimentos que simplificam a busca por soluções em domínios que são pouco compreendidos - de modo que a definição de uma função heurística possa levar a uma solução satisfatória sem comprometimento de processos decisórios; e padrões estocásticos como aqueles que têm origem em processos não determinísticos, com origem em eventos aleatórios e que permitem enunciar probabilidades - padrões esses utilizados pelo Kaipira.

O Kaipira constitui-se enquanto plataforma de "otimização multi-objetiva" contínua. Para tanto, trabalha com três módulos, EE, QFD e LF, possibilitando a seleção e orientação das variáveis a serem otimizadas no projeto, bem como a avaliação e classificação, pelo usuário, da importância das necessidades do projeto. Posteriormente, a aplicação do AG possibilita a otimização ponderada de uma configuração inicial. O algoritmo genético, método probabilístico multiobjetivo e multidisciplinar que apresenta boa varredura do domínio de soluções dos problemas considerados, é utilizado pelo Kaipira considerando 5 etapas: 1. Codificação, criação da população e cromossomos, pela qual se buscam as soluções com maior aptidão (cada grupo de soluções é entendido como "populações" geradas, traduzidas em número de cromossomos e sequências genéticas com as quais se deseja trabalhar, que podem ser redefinidas por meio de iteração); 2. Seleção e Reprodução, referente ao número de reproduções que se deseja realizar entre as populações geradas; 3. Cruzamento, etapa onde as populações são cruzadas e é feita a recombinação e troca aleatória entre o material genético pertencente aos casais selecionados (crossover). 4. Mutação, etapa que, a partir dos cruzamentos, permite a recombinação das características mais promissoras do projeto (sempre a critério do projetista), com a geração de uma nova população e o consequente desenvolvimento de uma nova simulação do projeto, uma vez que o número de gerações a ser trabalhado é indeterminado (cabe ao projetista defini-lo);

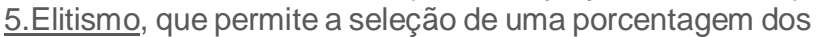
melhores indivíduos de uma geração de forma a manter uma boa herança genética e evitar a perda de estrutura de alta aptidão em gerações posteriores. Este último módulo do Kaipira apresenta interface com software de CAD Intercad 3D - e modelador de sólidos tridimensional - Solid Works (ver Fig. 3). 


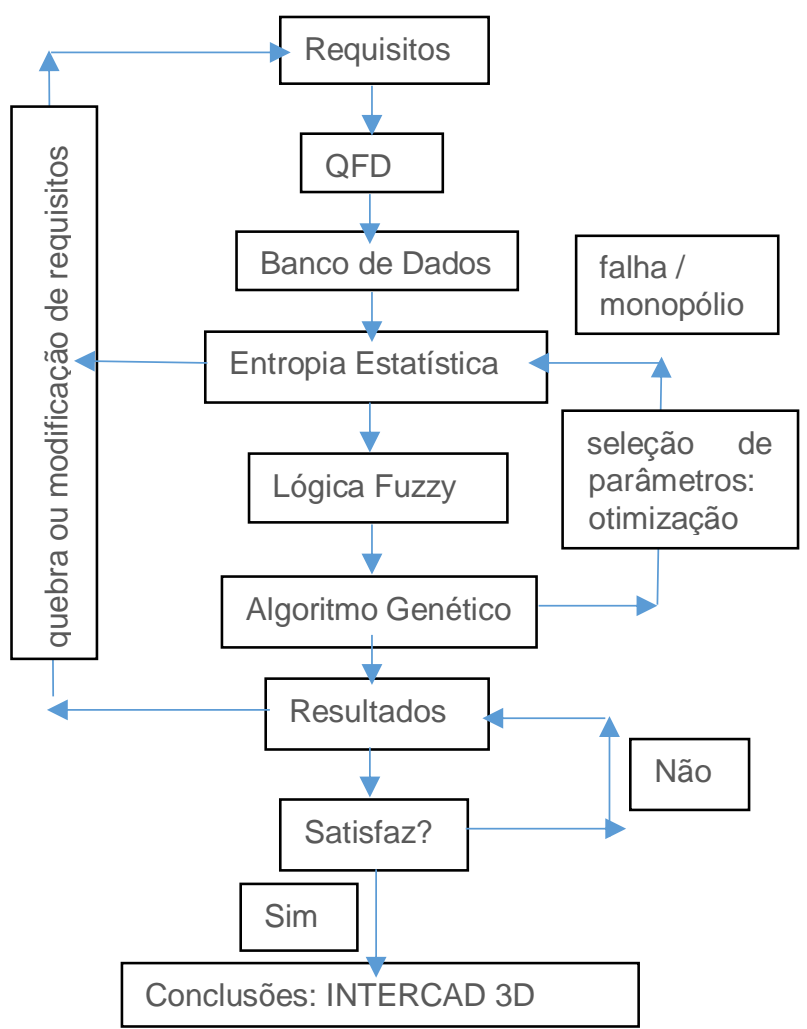

Figura 3: Fluxograma do Caipira.

Após definição do Plano de Massas, a utilização do Kaipira deu-se conforme variáveis e parâmetros urbanísticos, respectivamente: largura, comprimento, raio e altura da edificação; coeficiente de aproveitamento e taxas de ocupação e permeabilidade (ver Figs. 4 e 5).

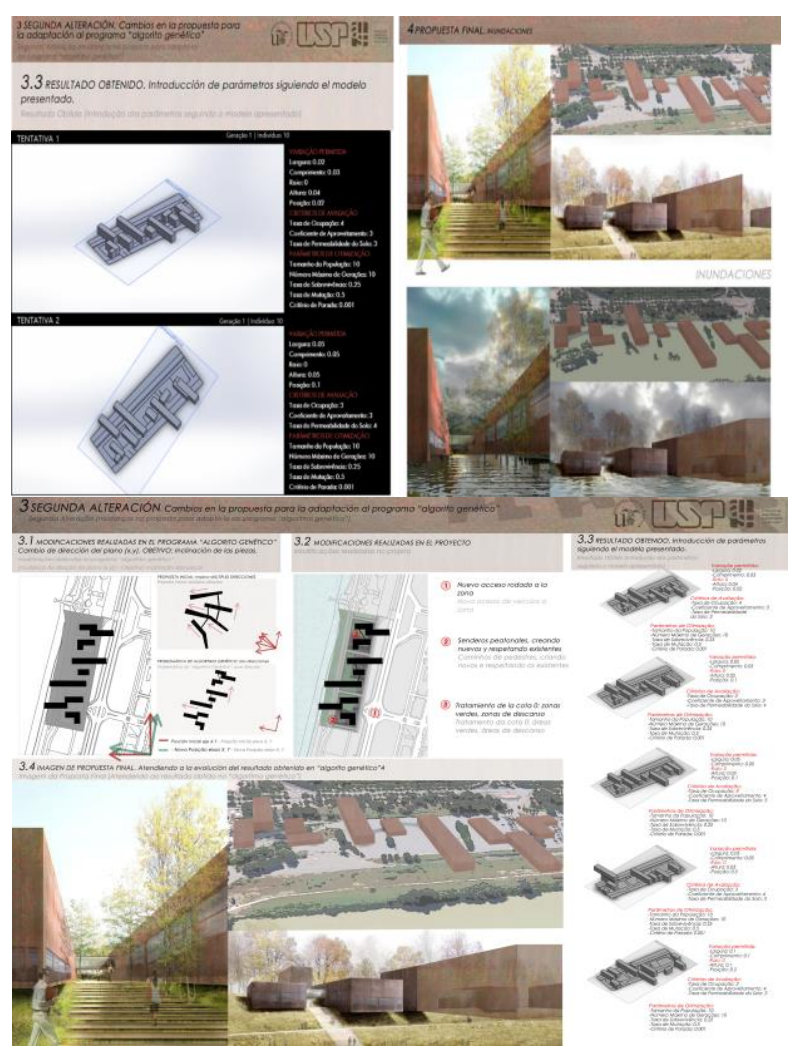

Figuras 4 e 5: Propostas finais, em que se observa o emprego de argumentos projetuais e do modelador de sólidos .

\section{RESULTADOS E CONCLUSÕES}

O workshop "Geração de Padrões Urbanos de Conexão", que pode ser qualificado como experimental, expressando um pensamento projetual singular decorrente do emprego de técnicas projetuais inovadoras, possibilitou não apenas a prática de metodologias didáticas não usuais, mas também o refinamento de ferramentas projetuais e a experimentação com trabalho colaborativo a distância.

Os processos de experimentação projetual compreenderam três fases: etapa 1, formulação de requisitos e especificações de projeto relativos a critérios e variáveis arquitetônicas; etapa 2, conceitual, de avaliação dos parâmetros empregados no algoritmo genético para o desenvolvimento do projeto; etapa 3, análise das soluções e de definição das diretrizes de desenvolvimento das soluções arquitetônicas.

As práticas e experimentações do workshop permitiram comprovar que o ferramental computacional empregado constitui um importante instrumento de gerenciamento do conhecimento e possibilita o desenvolvimento de novas abordagens para a formação de um arquiteto. Independentemente das dificuldades na troca de conteúdos teóricos entre cursos distintos, tanto por questões do projeto político-pedagógico de cada curso ou em função do fuso horário, os resultados apresentados foram muito satisfatórios em vários aspectos, mais precisamente: no desenvolvimento de um pensamento integrado para o projeto arquitetônico, tanto pelo uso de técnicas e procedimentos projetuais não usuais e inovadores, como pela troca de conteúdos em ambiente de característica transdisciplinar; no feedback para o aperfeiçoamento de ferramentas e técnicas projetuais experimentais; na prática de metodologias de ensino não ordinárias para atividades projetuais; na experimentação com o trabalho colaborativo a distância; e no crescimento e consolidação de intercâmbio acadêmico. Além disso, apesar das dificuldades decorrentes de agendas distintas, a proposta de agrupar os estudantes em grupos mistos, que combinavam membros da ETSA-US e do IAU-USP, mostrou-se acertada possibilitando uma troca acadêmica que enriqueceu as decisões de projeto, uma vez que as mesmas estavam sempre submetidas a critérios e parâmetros urbanísticos na análise de resultados.

Todavia, se por um lado Tecnologias de Informação e Comunicação (TICs) e técnicas de Inteligência Artificial (IA) abrem novas possibilidades para o desenvolvimento de conteúdos técnicos, teóricos e científicos e potencializam a interação entre diferentes agentes, por outro, o emprego dessas técnicas demonstram a necessidade de investigação de questões que permanecem por responder.

\section{DISCUSSÃO}

Portanto, restaria aqui, independentemente da necessidade de explicações mais prolixas deste breve texto, deixar emergir algumas dúvidas próprias quanto: ao processo de implementação de métodos de ensino / aprendizagem, presenciais e não-presenciais, que contemplem comunicações síncronas e assíncronas; e ao papel das TICs e de técnicas de Inteligência Artificial na educação arquitetônica.

O workshop buscou, por um lado, superar limites e dificuldades do ensino de projeto (fatos) e, por outro, 
explorar potencialidades (prospecção). A continuidade do trabalho compreende o aprimoramento da interface com modeladores de sólidos e o refinamento de estudos de desempenho de massa e volumetria (ver Figs. 6 e 7), de modo a possibilitar o refinamento da modelação paramétrica e, eventualmente, a prototipagem rápida.
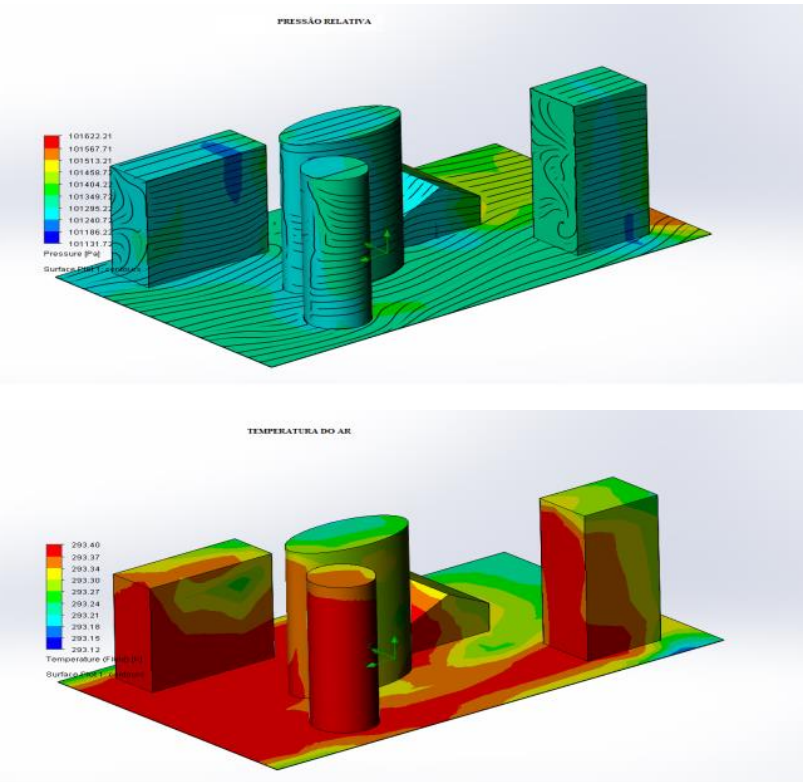

Figuras 6 e 7: Ensaios de Pressão e Temperatura de propostas em desenvolvimento.

A experiência do workshop "Geração de Padrões Urbanos de Conexão", teve desdobramentos em duas outras disciplinas: 'Reversos da Cidade: imaginários e tensões da cidade contemporânea' (graduação) e 'Cidade Virtual como Cena: o habitar de um ensaio' (pós-graduação) que, em maior ou menor grau, deram continuidade a exploração de práticas pedagógicas e do emprego de ferramental computacional na investigação da cidade contemporânea. Cabe aqui observar que essa investigação se baseia em um mínimo de pressupostos, de modo que própria reflexão que marca o desenvolvimento de conteúdos gera sua própria crítica, reconhecendo assim a complexidade da cidade e possibilitando distintas abordagens de sua problemática.

Assumindo o desafio de outras condições de contorno e deliberação em quanto a posições acadêmicas e professionais relativas a aspectos do crescimento e requalificação urbana, essas experiencias tem buscado extrapolar controvérsias de interesse no diagnóstico do presente, como o sentido político antidialético, a matéria e autenticidade arquitetônica, sua historicidade ou a questão da realidade, mediante o emprego de marcos de diferentes campos do conhecimento.

\section{REFERÊNCIAS}

Architectural Design (2009). Digital Cities, vol 79, num 04, JulyAugust 2009

Barner.M., Cottineau, C. (2017) Multiscale Entropy in the Spatial Context of Cities. Center for Advanced Spatial Analysis. London: University College, p.1-12.

Bauman, Z., Nuñez, V. (2007). Los Retos de la Educación en la Modernidad Líquida. Barcelona: Gedisa.

Bergström, A. (2014). Architecture And The Rise Of Practice In Education. Architectural Theory Review, 19(1), 10-21.
Blanco, L. M. (1993) La Arquitectura Cortes y otros Escritos. Madrid: UPM Editora.

Boeing, G. (2018) Measuring the Complexity of Urban Form and Design. Department of City and Regional Planning. Berkley: University of California (em publicação).

Chambers, D., Haley, K., Balata, F. (2017). Wherever you fiind people: the radical schools of Oscar Niemeyer, Darcy Ribeiro and Leonel Brizola. Zurich: Park Books.

Cuff, D. (1991). Architecture: the story of practice. Cambridge: MIT Press.

Deamer, P. (2012). Education of an Architect: a point of view and education of an architect Cooper Union, Journal of Architectural Education, p. 135-147.

Deb, K. (2001). Multi-Objective Optimization Using Evolutionary Algorithms. Chichester: John Willey \& Sons.

Déotte, J. L. (2013). La Ciudad Porosa: Walter Benjamin y la Arquitectura. Santiago do Chile: Metales Pesados.

Dutton, T. A. (ed.) (1991). Voices in architectural education: cultural politics and pedagogy. New York: Bergin \& Garvey.

Goldberg, D. E. (1989). Genetic algorithms in search, optimization, and machine learning. London: Addison Wesley Publishing Company, Inc.

Gutman, R., Bell, B., Cuff, D, Wriedt, J. (2010). Architecture from the outside in selected essays. New York: Princeton Architectural Press.

Hedjuk, J. (1995) Architectures in Love. New York: Rizzoli Press.

Kelbaugh, Douglas; McCullough, K. K. (ed.) (2014). Writing Urbanism: A Design Reader (The ACSA Architectural Education Series). New York: Routledge, Taylor \& Francis Group.

Lam, K.C., Tang, C.M., Lee, W.C. (2003) Application of the entropy technique and genetic algorithms to construction site layout planning of medium-size projects. Department of Building and Construction, City University Hong Kong.

Max-Neef, M. A. (2005). Fundamentos de la transdisciplinaridad. Ecological Economics, (53), 5-16.

Mitásová, M. (ed.) (2014). (Allen, S., Colomina, B., Frampton, K., Hays, M., McLeod, M., Ocman, J., Wigley, M. et al). Oxymoron and Pleonasm: conversations on american critical and projective theory of architetcure. Prague: Zlaty Fez.

Morin, E. (2011). Los Siete Saberes Necesarios para la Educación del Futuro. Barcelona: Paidós.

Nicolescu, B. (2013). La Necesidad de la Transdisciplinaridad en la Educación Superior. Trans-Pasando Fronteras, vol 3, num 03, p. 23-30.

Pallamin, V. (2007). Sobre o Ensino e Aprendizagem de Arquitetura e Urbanismo: as lições de $O$ Mestre Ignorante. Revista Pós, FAU-USP, São Paulo, p. 52-60.

Rancière, J. (2003). El Maestro Ignorante: cinco lecciones sobre la emancipación intelectual. Barcelona: Editorial Laertes.

Schumacher, P. (2009). Paremetricism: a new global style for architecture and urban design. Digital Cities, vol 79, num 04, july-august 2009, p. 14-23.

Sloterdijk, P. (2015). Los Hijos Terribles de la Edad Moderna: sobre el experimento antigenealógico de la modernidad. Madrid; Siruela.

Solà-Morales, M. (1997). Las Formas de Crecimiento Urbano. Barcelona: Edicciones UPC.

Spiridonidis, C., Voyatzak, M. (ed.) (2014). Learning for the Future. New Priorities of Schools of Architecture in the Era of Uncertainty. In ENHSA, 16th Meeting of Heads of European Schools of Architecture Dealing with Change for a Dynamic, Responsive, Adapatative and Engaged Architectural Education. Lifelong Learning Programme.

Su, Z., Yan, W. (2015) A Fast Generic Algorithm for Solving Architectural Design Optimization Problems. Artificial Intelligence for Engineering Design, Analysis and Manufacturing, 29, p. 457-469. Cambridge University Press.

Verebes, T. (2009). Experiments in Associative Urbanism. Digital Cities, vol 79, num 04, july-august 2009, p. 24-33. 